\title{
Efficacy of everolimus in ABO-incompatible kidney transplantation: a retrospective study
}

This article was published in the following Dove Press journal:

Transplant Research and Risk Management

27 September 2017

Number of times this article has been viewed

\author{
Kazuma Tsujimura' \\ Morihito Ota' \\ Kiyoshi Chinen' \\ Kiyomitsu Nagayama² \\ Masato Oroku² \\ Morikuni Nishihira ${ }^{2}$ \\ Yoshiki Shiohira ${ }^{2}$ \\ Kunitoshi Iseki ${ }^{3}$ \\ Hideki Ishida ${ }^{4}$ \\ Kazunari Tanabe 4 \\ 'Department of Surgery, ${ }^{2}$ Department \\ of Nephrology, ${ }^{3}$ Clinical Research \\ Support Center, Tomishiro Central \\ Hospital, Okinawa, Japan; ${ }^{4}$ Department \\ of Urology, Tokyo Women's Medical \\ University, Tokyo, Japan
}

Background: There are limited reports on the use of everolimus for maintaining immunosuppression in ABO-incompatible (ABOi) kidney transplantation (KT). As everolimus (EVR) is effective for preventing calcineurin inhibitor (CNI) nephrotoxicity without increasing the risk of chronic rejection and viral infection, we aimed to assess the efficacy of EVR in ABOi KT.

Patients and methods: We retrospectively studied 22 patients who underwent $\mathrm{KT}$ and received EVR. Patients in the ABOi KT group ( $n=7)$ were compared with those in the ABO-compatible kidney transplantation group (ABOc KT; $n=15$ ). We recorded the frequency of CNI nephrotoxicity, chronic rejection, and viral infection in the 2 groups.

Results: CNI nephrotoxicity, chronic rejection, and viral infection occurred in the ABOi KT and ANOc KT groups at rates of $28.3 \%$ (2/7 patients) and $13.3 \%(2 / 15$ patients) $(P=0.388)$, $28.3 \%$ (2/7 patients) and $26.7 \%$ (4/15 patients) $(P=0.926)$, and $14.3 \%(1 / 7$ patients $)$ and $26.7 \%$ (4/15 patients) $(P=0.517)$, respectively.

Conclusion: Administration of EVR is effective in preventing CNI nephrotoxicity after KT. The rate of CNI nephrotoxicity was lower in the ABOc KT group than in the ABOi KT group. The rate of chronic rejection and viral infection was comparable between the groups. This study was conducted in a small cohort of patients. Hence, further evaluation with large sample sizes is necessary in the future to confirm the outcomes.

Keywords: blood group incompatibility, immunosuppression, kidney transplantation

\section{Background}

In Japan, the number of deceased kidney transplantations (KTs) has decreased, while the number of living donor KTs has increased, and living donor KTs currently account for about $90 \%$ of all transplantations. Among living donor KTs, ABO-incompatible (ABOi) KTs have been widely performed, and they account for about $30 \%$ of living donor KTs. ${ }^{1}$ In the United States, 738 (0.94\%) ABOi KTs were performed between 1995 and 2010, and this number has been increasing annually. ${ }^{2}$ A similar trend has been observed in the United Kingdom, and over the last decade, there has been an increase in the number of ABOi KTs from $<10$ to 73 per year, currently representing about $7 \%$ of all living donor KTs. ${ }^{3}$ The number of ABOi KTs has been increasing worldwide, including in Japan.

After KT, immunosuppressive therapy with calcineurin inhibitors (CNIs) is extensively used as a maintenance regimen. Their use has decreased the acute rejection rate and has improved short-term patient survival and graft survival. ${ }^{4}$ However, in the long term, CNIs are associated with chronic graft dysfunction owing to CNI nephrotoxicity,
Correspondence: Kazuma Tsujimura Department of Surgery, Tomishiro Central Hospital, 25 Azaueda

Tomishiroshi, Okinawa, 901-0243, Japan

$\mathrm{Tel}+8 \mathrm{I} 9885038$ I I

Email tsujikazuma1978@yahoo.co.jp
Transplant Research and Risk Management 2017:9 43-48

43

Dovepress f 1 in $\bullet$

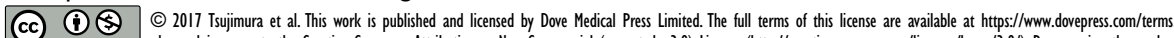

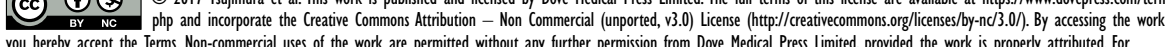

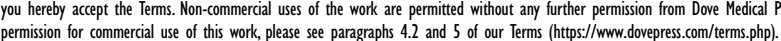


risks of malignancy, and cardiovascular issues. ${ }^{5,6} \mathrm{CNI}$ nephrotoxicity is a significant side effect because it can cause graft dysfunction. Everolimus (EVR) is commonly used in KTs in order to prevent the side effects of CNIs.

Previous literature reported ABOi KTs involving a de novo EVR-based regimen. ${ }^{7}$ However, there are limited reports on the use of EVR for maintenance immunosuppression in ABOi KTs. As EVR is effective for preventing CNI nephrotoxicity without increasing the risk of chronic rejection and viral infection, we aimed to assess the efficacy of EVR in ABOi KT.

\section{Patients and methods}

We retrospectively studied 22 patients who underwent KTs and received EVR between January 2013 and December 2015 at Tomishiro Central Hospital. Approval for the study was obtained from the Institutional Review Board of Tomishiro Central Hospital. All participants were given adequate verbal instructions, and they provided written informed consent.

In the induction phase, tacrolimus (TAC), mycophenolate mofetil (MMF), methylprednisolone (MP), and basiliximab (Basi) were administered in ABOi KTs and ABO-compatible (ABOc) KTs. In addition, rituximab (Rit) was administered and plasmapheresis was performed in ABOi and highly sensitive KTs. Oral administration of TAC, MMF, and MP was initiated 2 weeks before transplantation in ABOi KTs and 1 week before transplantation in ABOc KTs. Oral administration of
TAC was started at $0.1 \mathrm{mg} / \mathrm{kg} / \mathrm{d}$. The dose of TAC was adjusted to yield a trough concentration of $6-8 \mathrm{ng} / \mathrm{mL}$ within 1 month postoperatively, and then, the dose was reduced to maintain a target trough concentration of 5-7 ng/mL 3 months after KT. Administration of MMF was started at a dose of 1,000 $\mathrm{mg} / \mathrm{d}$, and the dose was increased to $2,000 \mathrm{mg} / \mathrm{d}$ from $\mathrm{KT}$ to 2 months after KT. The dose was then tapered to $1,000 \mathrm{mg} / \mathrm{d}$ at 3 months after KT. Administration of MP was started at a dose of $8 \mathrm{mg} / \mathrm{d}$, and the dose was changed to $250 \mathrm{mg} / \mathrm{d}$ on the day of KT. The dose was then reduced and maintained at $4 \mathrm{mg} / \mathrm{d}$ for 1 month postoperatively. Basi $(20 \mathrm{mg})$ was administered at KT and on postoperative day 4. Rit (200 mg) was administered 1 week before KT. The immunosuppressive regimen in our institution is summarized in Figure 1.

To standardize EVR administration, the following criteria were used: 1) the recipient did not have donor-specific antigens before KT; 2) the recipient did not have infection, uncontrollable hyperlipidemia, and proteinuria $(>800 \mathrm{mg} / \mathrm{d})$ after KT; 3) acute rejection was not observed on protocol biopsy 3 months after KT.

Furthermore, we reduced the TAC dose upon EVR initiation. The dose of TAC was tapered to maintain a target trough concentration of $1.5-3 \mathrm{ng} / \mathrm{mL}$, and the dose of EVR was adjusted to maintain a target trough concentration of 3-8 ng/mL.

EVR was administered in all 22 study patients after 3 months postoperatively. Patients in the ABOi KT group $(n=7)$

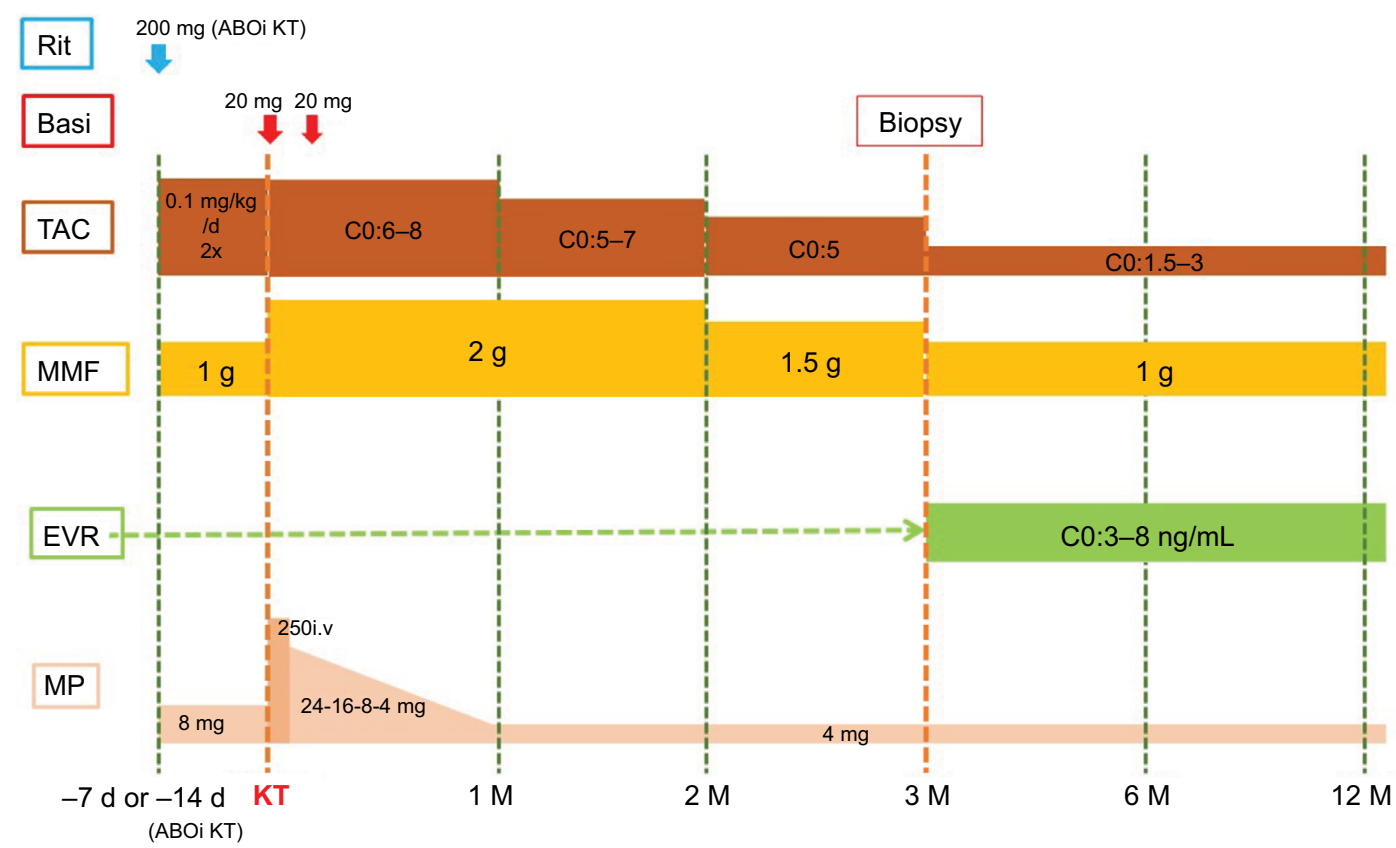

Figure I Immunosuppressive regimens and desensitization protocols.

Abbreviations: $\mathrm{ABO}$, ABO-incompatible; Basi, basiliximab; $\mathrm{C} 0$, trough concentration; EVR, everolimus; KT, kidney transplantation; MMF, mycophenolate mofetil; MP, methylprednisolone; Rit, rituximab; TAC, tacrolimus. 
were compared to those in the ABOc KT group ( $\mathrm{n}=15)$. We evaluated the serum creatinine (Scr) level, estimated glomerular filtration rate (eGFR), frequency of CNI nephrotoxicity, chronic rejection, viral infections, malignancy, and adverse events in the 2 groups. We calculated the eGFR using the Modification of Diet in Renal Disease formula.

According to our institutional policy, protocol biopsies were performed in all patients after obtaining written consent at 3 months after KT and every year thereafter. Additional biopsies were obtained when renal graft function was impaired or BK virus viremia developed. Renal biopsy samples were evaluated by a specialist nephropathologist. Chronic rejection and CNI nephrotoxicity were determined according to the diagnostic criteria proposed at the 2013 Banff Conference. ${ }^{19}$ We included borderline change for chronic rejection. CNI nephrotoxicity was defined a priori by the appearance of de novo arteriolar hyalinosis.

Cytomegalovirus (CMV) infection was assessed using the antigenemia method (C7-HRP) once every 2 weeks within 3 months of KT and once every month thereafter in all recipients. The diagnosis of BK polyomavirus (BKV) infection was based on the finding of viral DNA in the plasma using polymerase chain reaction and the presence of histologic features in combination with positive staining for SV40.
Hyperlipidemia was defined as the use and requirement of one or more hypolipidemic drugs. The definition of proteinuria positivity was a protein-to-creatinine $(\mathrm{Cr})$ ratio $\geq 150 \mathrm{mg} / \mathrm{gCr}$.

Data are expressed as mean \pm standard deviation (SD). For statistical analysis, the unpaired $t$-test and $\chi^{2}$ test were used to compare differences between the ABOi KT and ABOc KT groups. A $P$-value $<0.05$ was considered statistically significant. All statistical analyses were performed using JMP software, version 11.0 (SAS Institute, Inc., Cary, NC, USA).

\section{Results}

The characteristics of the patients in the ABOi KT and ABOc KT groups are presented in Table 1. The ABOi KT group included 5 men and 2 women (mean age, 49.0 \pm 7.1 years; Rit administration, 7 of 7 patients (100\%); mean EVR administration period, $31.3 \pm 9.6$ months; mean EVR dose, $4.21 \pm 0.90 \mathrm{ng} / \mathrm{mL})$. The ABOc KT group included 8 men and 7 women (mean age, $45.8 \pm 17.0$ years; Rit administration, 2 of 15 patients $(13.3 \%)$; mean EVR administration period, $27.1 \pm 10.3$ months; mean EVR dose, $4.77 \pm 1.51 \mathrm{ng} / \mathrm{mL}$ ). All patient characteristics, except for Rit administration, did not differ significantly between the 2 groups.

The outcomes of patients treated with EVR in the 2 groups are shown in Table 2. In the ABOi KT group, the mean

Table I Patient characteristics

\begin{tabular}{|c|c|c|c|}
\hline & ABOi KT $(n=7)$ & ABOc KT $(n=15)$ & $P$-value \\
\hline Male, n (\%) & $5(71.4 \%)$ & $8(53.3 \%)$ & 0.421 \\
\hline Age (years) & $49.0 \pm 7.1(40-57)$ & $45.8 \pm 17.0(13-67)$ & 0.640 \\
\hline Rit administration, $\mathrm{n}(\%)$ & $7(100 \%)$ & $2(13.3 \%)$ & $<0.001$ \\
\hline Period after KT (months) & $39.6 \pm 14.5(23-56)$ & $33.5 \pm 12.7(15-55)$ & 0.331 \\
\hline EVR administration period (months) & $31.3 \pm 9.6(|9-4|)$ & $27.1 \pm 10.3(12-42)$ & 0.372 \\
\hline TAC dose $(\mathrm{ng} / \mathrm{mL})$ before EVR administration & $4.96 \pm 1.92(3.5-9.1)$ & $5.23 \pm 1.30(3.1-7.5)$ & 0.702 \\
\hline TAC dose $(\mathrm{ng} / \mathrm{mL})$ after EVR administration & $1.67 \pm 0.45(1.1-2.2)$ & $1.8 \mathrm{I} \pm 0.64(\mathrm{I} . \mathrm{I}-3.7)$ & 0.608 \\
\hline EVR dose $(\mathrm{ng} / \mathrm{mL})$ & $4.2 \mathrm{I} \pm 0.90(3.2-5.3)$ & $4.77 \pm I .5 \mathrm{I}(3.0-7.5)$ & 0.385 \\
\hline
\end{tabular}

Note: Data are presented as mean \pm SD (range).

Abbreviations: $\mathrm{ABOc}$, $\mathrm{ABO}$-compatible; $\mathrm{ABOi}, \mathrm{ABO}$-incompatible; EVR, everolimus; $\mathrm{KT}$, kidney transplantation; Rit, rituximab; $\mathrm{SD}$, standard deviation; $\mathrm{TAC}$, tacrolimus.

Table 2 Outcome of patients treated with EVR

\begin{tabular}{|c|c|c|c|}
\hline & ABOi KT $(n=7)$ & ABOc KT $(n=15)$ & P-value \\
\hline Scr levels before EVR administration (mg/dL) & $1.20 \pm 0.29(0.84-1.55)$ & $1.33 \pm 0.54(0.64-2.19)$ & 0.559 \\
\hline Current Scr levels after EVR administration (mg/dL) & $1.25 \pm 0.39(0.75-1.80)$ & $1.28 \pm 0.54(0.63-2.20)$ & 0.894 \\
\hline eGFR before EVR administration $\left(\mathrm{mL} / \mathrm{min} / \mathrm{I} .73 \mathrm{~m}^{2}\right)$ & $62.9 \pm 19.0(47.5-99.8)$ & $60.9 \pm 23.9(25.1-102.3)$ & 0.847 \\
\hline eGFR after EVR administration $\left(\mathrm{mL} / \mathrm{min} / \mathrm{I} .73 \mathrm{~m}^{2}\right)$ & $63.6 \pm 26.8(30.8-87.8)$ & $63.7 \pm 23.9(28.0-115.3)$ & 0.995 \\
\hline CNI nephrotoxicity, n (\%) & $2(28.6 \%)$ & $2(13.3 \%)$ & 0.3881 \\
\hline Chronic rejection, $\mathrm{n}(\%)$ & $2(28.6 \%)$ & $4(26.7 \%)$ & 0.926 \\
\hline Viral infections, n (\%) & I (I4.3\%) & $4(26.7 \%)$ & 0.517 \\
\hline Malignancy, n (\%) & $0(0 \%)$ & $0(0 \%)$ & - \\
\hline
\end{tabular}

Note: Data are presented as mean \pm SD (range).

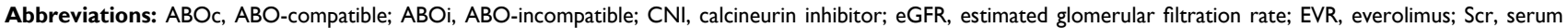
creatinine; SD, standard deviation. 
Scr levels and eGFR before and after EVR administration were $1.20 \pm 0.29 \mathrm{mg} / \mathrm{dL}$ and $1.25 \pm 0.39 \mathrm{mg} / \mathrm{dL}$, and $62.9 \pm 19.0$ and $63.6 \pm 26.8 \mathrm{~mL} / \mathrm{min} / 1.73 \mathrm{~m}^{2}$, respectively. In the ABOc KT group, the mean Scr levels and eGFR before and after EVR administration were $1.33 \pm 0.54 \mathrm{mg} / \mathrm{dL}$ and $1.28 \pm 0.54$ $\mathrm{mg} / \mathrm{dL}$, and $60.9 \pm 23.9$ and $63.7 \pm 23.9 \mathrm{~mL} / \mathrm{min} / 1.73 \mathrm{~m}^{2}$, respectively. The mean Scr levels and eGFR did not differ significantly before and after EVR administration in the 2 groups. Graft survival rate was $100 \%$ in both groups. CNI nephrotoxicity occurred in 2 of 7 patients $(28.3 \%)$ in the ABOi KT group and 2 of 15 patients (13.3\%) in the ABOc KT group $(P=0.388)$. Chronic rejection occurred in 2 of 7 patients $(28.3 \%)$ in the ABOi KT group and in 4 of 15 patients $(26.7 \%)$ in the ABOc KT group $(P=0.926)$. Viral infections occurred in 1 of 7 patients (14.3\%) in the ABOi KT group and in 4 of 15 patients $(26.7 \%)$ in the ABOc KT group $(P=0.517)$. With regard to viral infections, in the $\mathrm{ABOi} \mathrm{KT}$ group, $\mathrm{BKV}$ infection occurred in only 1 patient, and in the ABOc KT group, herpes simplex virus (HSV) and varicella-zoster virus (VZV) infections occurred in 1 and 4 patients, respectively (1 patient had multiple infections). Malignancy was not noted in any patient from the 2 groups.

Adverse events that occurred with EVR in the 2 groups are shown in Table 3. Hyperlipidemia that required statin drug administration was the most common adverse event of EVR and occurred in 4 of 7 patients $(57.1 \%)$ in the ABOi KT group and 11 of 15 patients $(73.3 \%)$ in the ABOc KT group $(P=0.448)$. With regard to other adverse events, pedal edema, stomatitis, and proteinuria occurred in the 2 groups. However, severe adverse events did not occur in the 2 groups.

\section{Discussion}

There is sufficient evidence to indicate that a low-dose CNI with an mTOR inhibitor is safe and effective in the long term. ${ }^{8}$ We started to administer EVR in our institution in accordance with previously mentioned criteria in ABOi and ABOc KTs. As our institution had limited experience with the use of EVR, recipients selected based on the criteria had

Table 3 Adverse events occurring with EVR in the 2 groups

\begin{tabular}{llll}
\hline & $\begin{array}{l}\text { ABOi KT } \\
(\mathbf{n}=7)\end{array}$ & $\begin{array}{l}\text { ABOc KT } \\
(\mathbf{n}=15)\end{array}$ & P-value \\
\hline Hyperlipidemia, n (\%) & $4(57.1 \%)$ & $\mathrm{II}(73.3 \%)$ & $P$-value \\
Pedal edema, n (\%) & $\mathrm{I}(14.3 \%)$ & $6(40.0 \%)$ & $P$-value \\
Stomatitis, n (\%) & $5(71.4 \%)$ & $6(40.0 \%)$ & $P$-value \\
Proteinuria, n (\%) & $0(0 \%)$ & $3(20 \%)$ & $P$-value \\
\hline
\end{tabular}

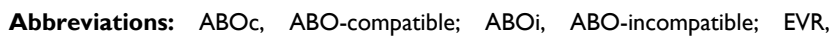
everolimus; $\mathrm{KT}$, kidney transplantation. a low risk of acute rejection and low risk of adverse events of EVR, such as hyperlipidemia and proteinuria.

In Japan, the 10-year graft survival rates for deceased donor transplants were $51.4 \%$ and $71.1 \%$ from 1983 to 2000 and 2001 to 2009 , respectively. The 10 -year graft survival rates for living donor transplants were $69.1 \%$ and $87.0 \%$ from 1983 to 2000 and 2001 to 2009 , respectively. ${ }^{1}$ In the United States, among deceased donor kidney transplant recipients, the probability of all-cause graft failure at 10 years after transplantation decreased from 59\% in 1996 to 55\% in 2003. Similarly, for living donor kidney transplant recipients, the probability of all-cause graft failure at 10 years after transplantation decreased from $43 \%$ in 1996 to $40 \%$ in $2003 .{ }^{9}$ Globally, graft survival has continued to improve in recent years. It is very important to avoid graft dysfunction after $\mathrm{KT}$ in the long term. Accordingly, administration of EVR is useful to avoid CNI nephrotoxicity associated with chronic graft dysfunction after KT. In this study, CNI nephrotoxicity occurred in 2 of 7 patients $(28.3 \%)$ in the ABOi KT group and 2 of 15 patients (13.3\%) in the ABOc group. The rate of CNI nephrotoxicity tended to be lower in the ABOc KT group than in the ABOi KT group; however, the rate of CNI nephrotoxicity did not differ significantly between the groups $(P=0.388)$. Additionally, the Scr level after EVR administration did not differ between the ABOi KT group and ABOc $\mathrm{KT}$ group $(P=0.894)$. We consider that administration of EVR is more useful in ABOi KT than in ABOc KT for 2 reasons.

First, we consider that administration of EVR after ABOi KT prevents viral infection. The risk of infection is higher in ABOi KT patients than in ABOc KT patients. In ABOi KT, generally, Rit (anti-CD20 antibody) is administered or splenectomy and plasmapheresis are performed. Therefore, immunosuppression is often more intense in ABOi KT patients than in ABOc KT patients. Habicht et a ${ }^{10}$ confirmed a significantly higher rate of viral infections in ABOi KT patients than in ABOc KT patients. The rate of viral infections, including CMV, HSV, VZV, and BKV, have been reported to be significantly higher in the ABOi KT group (50\%) than in the ABOc KT group $(21 \%)(P=0.038)$. In the present study, the rate of viral infections, including CMV, HSV, VZV, and BKV, in the ABOi KT and ABOc KT groups was $14.3 \%$ and $26.7 \%$, respectively. The rate of viral infection did not differ between the groups; however, the rate tended to be lower in the ABOi KT group than in the ABOc KT group. The rates of viral infection among the $\mathrm{ABOi} \mathrm{KT}$ and $\mathrm{ABOc} \mathrm{KT}$ patients in the present study were lower than the rates among recipients who did not receive EVR in previous studies. 
Second, we consider that the risk of malignancy is higher in ABOi KT patients than in ABOc KT patients. In a previous multivariate analysis of the Organ Procurement and Transplantation Network database, the incidence of posttransplant malignancies was assessed in 33,249 patients. ${ }^{11}$ The results showed that the incidence of de novo malignancies was lower in patients receiving sirolimus or EVR, either alone or in combination with a CNI, than in patients receiving $\mathrm{CNI}$ therapy alone $(P<0.05) .{ }^{11}$ Yamamoto et a ${ }^{12}$ compared the risk of malignancy between ABOi KT and $\mathrm{ABOc} \mathrm{KT}$ and found that there was no significant difference in the incidence of malignancy between ABOi KT and ABOc KT (4.8\% and 4.2\%). The median follow-up time was 48 months. Similarly, Hall et a $1^{13}$ compared the risk of malignancy between ABOi KT and ABOc KT. The authors found that there was no difference in the overall cancer risk between $\mathrm{ABOi} \mathrm{KT}$ and $\mathrm{ABOc} \mathrm{KT}$ when considering the unadjusted incidence rate ratio $(0.83 \%)$ or the matched incidence rate ratio $(0.99 \%)$ analysis. The median follow-up time was 2 years. In these reports, there was no significant difference in the incidence of malignancy between ABOi KT and ABOc KT. However, further research with long-term follow-up is necessary, to adequately assess the risk of malignancy in ABOi KT. The current graft survival term is relatively long in ABOi KT patients. A previous study reported that the 9-year graft survival rate in ABOi KT patients was $86.9 \%$ from 2005 to $2013 .{ }^{14}$ The graft survival time in $\mathrm{ABOi} \mathrm{KT}$ patients might continue to increase. Therefore, in the long term, it is important that we consider the risk of malignancy in ABOi KT. In this study, there was no case of malignancy in the ABOi KT and ABOc KT groups. Because the observation period was short, we should continue to check for the occurrence of malignancy in transplant recipients in the future.

Among the adverse events of EVR, one adverse event that occurs with sirolimus and EVR treatment is hyperlipidemia, and increased serum cholesterol and triglyceride levels have been reported in $30 \%-50 \%$ of patients. ${ }^{15}$ In the present study, the rates of hyperlipidemia in the ABOi KT and ABOc KT groups were $57.1 \%$ and $73.3 \%$, respectively. The rate of hyperlipidemia was higher in the present study than in previous reports, and it could be controlled with statin drugs. With regard to other adverse events, pedal edema and stomatitis occurred at a relatively high rate. However, severe adverse events of EVR did not occur in the 2 groups.

Recipients in the ABOi KT and ABOc KT groups started treatment with EVR at a mean of 8 and 6 months, respectively, in the present study. The time at which EVR treatment should be started is not clear. In a previous study, recipients who started treatment with EVR within 12 months after transplantation showed an improvement in the eGFR at 12 months compared to the eGFR at baseline. ${ }^{16}$ It is known that in desensitized patients, surgical complications, especially wound healing complications, are more common. ${ }^{10,17,18}$ If recipients start treatment with EVR before transplantation and immediately after transplantation, wound healing complications might become a problem. Therefore, treatment with EVR should be started within 6-12 months.

\section{Conclusion}

Administration of EVR is effective in preventing CNI nephrotoxicity after KT. The rate of CNI nephrotoxicity was lower in the ABOc KT group than in the ABOi KT group. The rate of chronic rejection and viral infection was comparable in both groups. This study was conducted on a small cohort. Hence, we need further evaluation and confirmation with a large sample size in the future.

\section{Acknowledgments}

We thank Ms. Shoko Nakaema for providing valuable advice and support. We also thank Editage (www.editage.jp) for English language editing.

\section{Disclosure}

The authors report no conflicts of interest in this work.

\section{References}

1. The Japan Society for Transplantation, Japanese Society for Clinical Renal Transplantation. Annual progress report from the Japanese Renal Transplant Registry: number of renal transplantation in 2015 and followup survey. Jpn J Transplant. 2016;51:124-144.

2. Montgomery JR, Berger JC, Warren DS, James NT, Montgomery RA, Segev DL. Outcomes of ABO-incompatible kidney transplantation in the United States. Transplantation. 2012;93:603-609.

3. Organ Donation and Transplantation Activity Report; 2014. Available from http://nhsbtmediaservices.blob.core.windows.net/organ-donationassets/pdfs/activity_report_2014_15.pdf. Accessed September 25, 2016.

4. Sahin S, Gürkan A, Uyar M, et al. Conversion to proliferation signal inhibitors-based immunosuppressive regimen in kidney transplantation: to whom and when? Transplant Proc. 2011;43:837-840.

5. Guerra G, Srinivas TR, Meier-Kriesche HU. Calcineurin inhibitorfree immunosuppression in kidney transplantation. Transplant Int. 2007;20:813-827.

6. Hernández D, Martínez D, Gutiérrez E, et al. Clinical evidence on the use of anti-mTOR drugs in renal transplantation. Nefrologia. 2011;31:27-34.

7. Koch M, Wiech T, Marget M, et al. De novo mTOR inhibitor-based immunosuppression in ABO-incompatible kidney transplantation. Clin transplant. 2015;29:1021-1028.

8. Webster AC, Lee VW, Chapman JR, Craig JC. Target of rapamycin inhibitors (sirolimus and everolimus) for primary immunosuppression of kidney transplant recipients: a systematic review and meta-analysis of randomized trials. Transplantation. 2006;81:1234-1248.

9. The 2015 Annual Date Report. Available from https://www.usrds org/2015/view/v2_07.aspx. Accessed October 9, 2016. 
10. Habicht $A$, Bröker V, Blume C, et al. Increase of infectious complications in ABO-incompatible kidney transplant recipients - a single centre experience. Nephrol Dial Transplant. 2011;26:4124-4131.

11. Kauffman HM, Cherikh WS, Cheng Y, Hanto DW, Kahan BD. Maintenance immunosuppression with target-of-rapamycin inhibitors is associated with a reduced incidence of de novo malignancies. Transplantation. 2005;80:883-889.

12. Yamamoto T, Kawaguchi T, Watarai Y, et al. Potent immunosuppression for ABO-incompatible renal transplantation may not be a risk factor for malignancy. Transplant Proc. 2012;44:210-213.

13. Hall EC, Engels EA, Montgomery RA, Segev DL. Cancer risk following ABO incompatible living donor kidney transplantation. Transplantation. 2013;96:476.

14. Okumi M, Toki D, Nozaki T, et al. ABO-incompatible living kidney transplants: evolution of outcomes and immunosuppressive management. Am J Transplant. 2016;16:886-896.
15. Pascual J, Boletis IN, Campistol JM. Everolimus (Certican) in renal transplantation: a review of clinical trial data, current usage, and future directions. Transplant Rev. 2006;20:1-18.

16. Cicora F, Massari, P, Acosta F, et al. Use of everolimus in rena 1 transplant recipients: data from a national registry. Transplant Proc. 2014;46:2991-2995.

17. Koch M, Kantas A, Ramcke K, Drabik AI, Nashan B. Surgical complications after kidney transplantation: different impacts of immunosuppression, graft function, patient variables, and surgical performance. Clin Transplant. 2015;29:252-260.

18. Kuo JH, Wong MS, Perez RV, Li CS, Lin TC, Troppmann C. Renal transplant wound complications in the modern era of obesity. J Surg Res. 2012;173:216-223.

19. Haas M, Sis B, Racusen LC, et al. Banff 2013 meeting report: Inclusion of c4d-negative antibody-mediated rejection and antibody-associated arterial lesions. Am J Transplant. 2014;14:272-283.
Transplant Research and Risk Management

\section{Publish your work in this journal}

Transplant Research and Risk Management is an international, peerreviewed open access journal focusing on all aspects of transplantation and risk management to achieve optimal outcomes in the recipient improving survival and quality of life. The manuscript management system is completely online and includes a very quick and fair peer-review system,

\section{Dovepress}

which is all easy to use. Visit http://www.dovepress.com/testimonials.php to read real quotes from published authors. 